$$
\text { CONF-96077.3-4 SANO96-1858 }
$$

\title{
Space Applications of the MITS Electron-Photon Monte Carlo Transport Code System
}

\author{
Ronald P. Kensek, Leonard J. Lorence, John A. Halbleib \\ Sandia National Laboratories \\ Albuquerque, NM 87185-1179
}

\author{
J. E. Morel \\ Los Alamos National Laboratory \\ Los Alamos, NM 87545
}

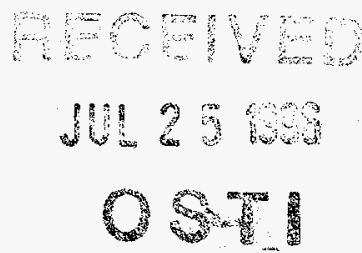

\begin{abstract}
The MITS multigroup/continuous-energy electron-photon Monte Carlo transport code system has matured to the point that it is capable of addressing more realistic three-dimensional adjoint applications. It is first employed to efficiently predict point doses as a function of source energy for simple threedimensional experimental geometries exposed to simulated uniform isotropic planar sources of monoenergetic electrons up to $4.0 \mathrm{MeV}$. Results are in very good agreement with experimental data. It is then used to efficiently simulate dose to a detector in a subsystem of a GPS satellite due to its patural electron environment, employing a relatively complex model of the satellite. The capability for survivability analysis of space systems is demonstrated, and results are obtained with and without variance reduction.
\end{abstract}

\section{INTRODUCTION}

The adjoint transport method is a powerful technique for simulating applications where a knowledge of the particle flux is only required for a restricted region of phase space, but where this knowledge is required for source parameters spanning a large region of phase space. Space applications often fall into this category. For example, mission-averaged radiation environments encountered by space systems are often broadly distributed in space, energy, and angle. Furthermore, the analyst may only be interested in the effect of this radiation on a small region of the system, such as the dose to a small detector.

In the important case of coupled electron photon transpiort, the only method that meets the combined requirements of space applications for geometrical complexity and physical accuracy is Monte Carlo. The Integrated TIGER Series (ITS) [1] is one of a few codes that meets these requirements. However, the nun times for the simulation of many of these applications using conventional, or forward, transport like that of ITS are so probibitive that the adjoint method may be the only practical method for solving such problems.

We have developed an adjoint capability for ITS that should be especially suited to space applications. This Multigroup/
Continuous-Energy Integrated TIGER Series (MTTS) Monte Carlo code system [2] combines the cross section generator from the CEPXS/ONELD code system [3] with the input/output and geometry routines of ITS to obtain the Monte Carlo solution of the time-independent Boltzmann-Fokker-Planck equation [4]. A preliminary version of the MITS system has been extensively verified against experiment and other computational methods in one dimensional applications, and has also been verified against other Monte Cario methods in a simple three-dimensional benchmark [2]. In particular, the utility of the adjoint mode of MITS was demonstrated. The software has matured to the point where more realistic applications can be addressed.

In this paper we first compare adjoint predictions of MITS with experimental data for a relatively simple threedimensional application. In these experiments the spatial and angular distribution of the source simulate those that are characteristic of approximately uniform isotropic radiation fields often encountered in space applications. In an application of much greater geometrical complexity, the MTTS system is subsequently used, again in the adjoint mode, to predict the energy deposition in a subsystem of the Global Positioning System (GPS) satellites due to the natural electron environment. The adjoint method efficiently predicts the dependence of the deposition on the kinetic energy and spatial location of the source electrons. In particular, the latter demonstrates how the adjoint method can reveal weaknesses in the shielding of selected satellite components.

All calculations were performed on an IBM RS/6000 970 (560-equivalent) work station.

\section{3-D EXPERIMENTAL VERIFICATION}

Van Gunten [5,6] measured three-dimensional dose distributions in hollow aluminum cubes due to isotropic radiation fields of electrons of various monoenergetic sources. Using radiochromic-dye film dosimeters and a scheme for simulating the isotropic fields, he measured the dose distributions inside cubes of varying wall thicknesses at electron source energies up to $4.0 \mathrm{MeV}$. 


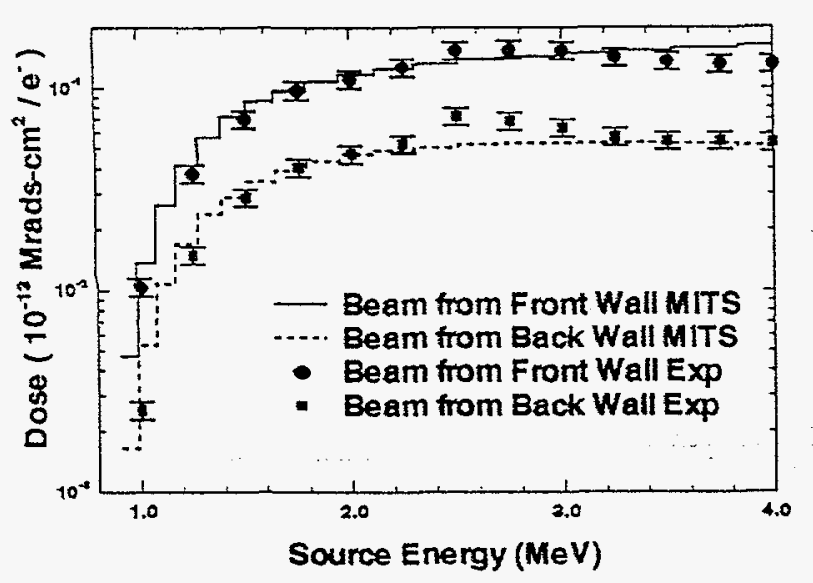

FIGURE 1. Comparison of the dose measurements of Van Gunten $[5,6]$ at the front and back wall of an aluminum cube irradiated on the front wall by a uniform, isotropic, and monoenergetic flux as a function of source electron energy with the predictions of a single adjoint calculation.

A single adjoint calculation was performed for the dose at the center and just inside a wall, where that wall is exposed to the electron source (front wall) and where the opposite wall is exposed to the source (back wall). By symmetry the same experimental data was obtained by irradiating only one wall. Figure 1 compares the calculated data with the experimental data. Not shown, but also obtained from this calculation, are the predicted doses where any of the four lateral walls are exposed to the source (these four distributions are the same from the symmetry of the problem). Thus, a single adjoint calculation gives the predicted dose at a point for all source energies and all source surfaces. Obtaining this information would have required multiple calculations in the forward mode. The agreement between tho calculations and the experimental data in Fig. 1 is quite good. The run time for the calculation was 4.7 hrs, resulting in a statistical uncertainty that is usually less than $1 \%$ but reaches a maximum of $3 \%$ in the lowest-energy group.

The edge of the aluminum cube is $20.32 \mathrm{~cm}$ and the wall thickness is $0.1524 \mathrm{~cm}$. The latter is small enough that the dose is dominated by direct electron deposition over the range of source energies plotted in Fig. 1. If lower energies or thicker walls had been chosen, the dose would have been dominated by bremsstrahlung produced by the source electrons. This would have been a more severe test of the MITS code, but experimental data for this situation were not available. An example of such a "combined radiation effects" adjoint calculation using the MITS system is given in the following GPS application (see also Reference [2]).

In contrast to Fig. 1, Fig. 2 shows the more familiar sort of information ottained from a single forward calculation at 3.0 $\mathrm{MeV}$, namely energy deposition profiles as a function of distance from the wall exposed to the source along a line through its center and one through its comer. Obtaining this information would have required multiple calculations in the adjoint mode. Agreement with experiment is again very good.

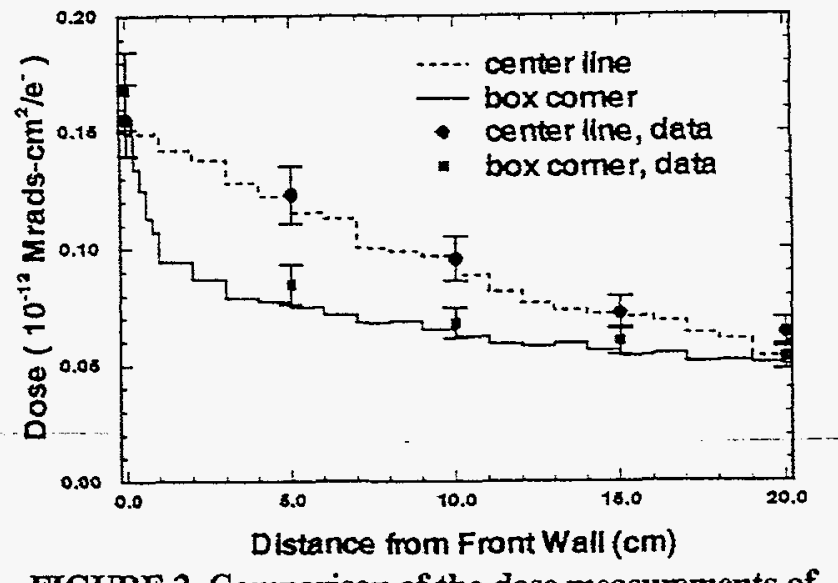

FIGURE 2. Comparison of the dose measurements of Van Gunten $[5,6]$ as a function of distance from the wall of an aluminum cube irradiated by 8 uniform, isotropic, 3.0 MeV flux along a line through the center and through the corner of the wall with the predictions of a single forward calculation.

Note also the agreement between tho forward calculation of the dose at the extremes of the profile along the center with the two adjoint results at $3.0 \mathrm{MeV}$ in Fig. 1.

\section{GPS APPLICATION}

Because of the altitude and inclination at which GPS satellites are deployed, they are exposed to a very severe natural electron environment. Components must be sufficiently radiation hard to survive the mission-averaged total dose. The MITS code system has the capability of predicting these doses with a higher degree of confidence than the less accurate masssectoring codes, albeit with a much longer run time. On the other hand, it is applications such as these for which simulation by conventional forward Monte Carlo codes like ITS is precluded by excessive ran times.

One of the subsystems aboard the GPS satellite is the Burst Detector Dosimeter (BDD) box. The ACCEPT code of the MITS systern was employed to calculate the dose at a point within this subsystem.

\section{A. Geometry}

Figure 3 is a projection of the geometrical model employed for radiation analysis of the BDD box. It consists of a set of components inside an (actual) aluminum box. The five external walls of the box are $0.508 \mathrm{~cm}$ thick. The model is described using the combinatorial geometry (CG) method of the ACCEPT code and represents all materials by aluminum with different mass densities so as to preserve the actual masses and dimensions. The model includes an artificial array of small hemispherical shells surrounding the locations of the detectors [7] where dose is to be calculated. The detector location selected for the adjoint MITS simulation is indicated in the figure. The hemispherical shell had inner and outer radii of 0.1067 and $0.1270 \mathrm{~cm}$, respectively. 


\section{DISCLAIMER}

Portions of this document may be illegible in electronic image products. Images are produced from the best available original document. 



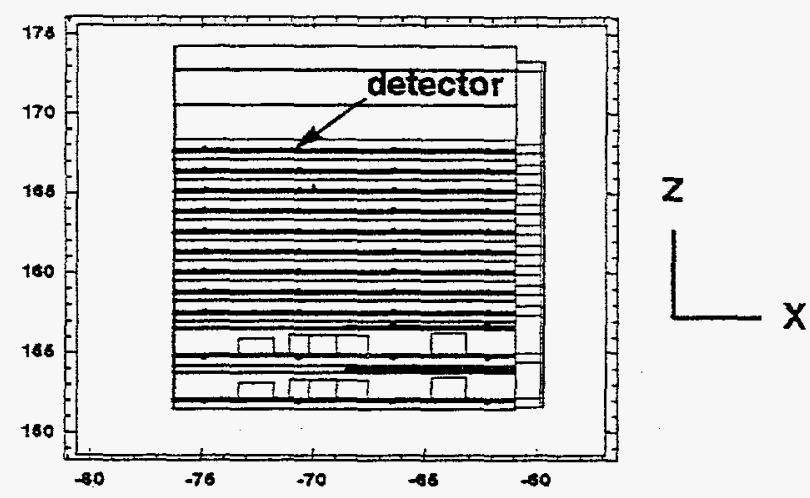

FIGURE 3. Projection of the geometrical model of the BDD box employed in calculations with the MITS code of the dose at the indicated detector.

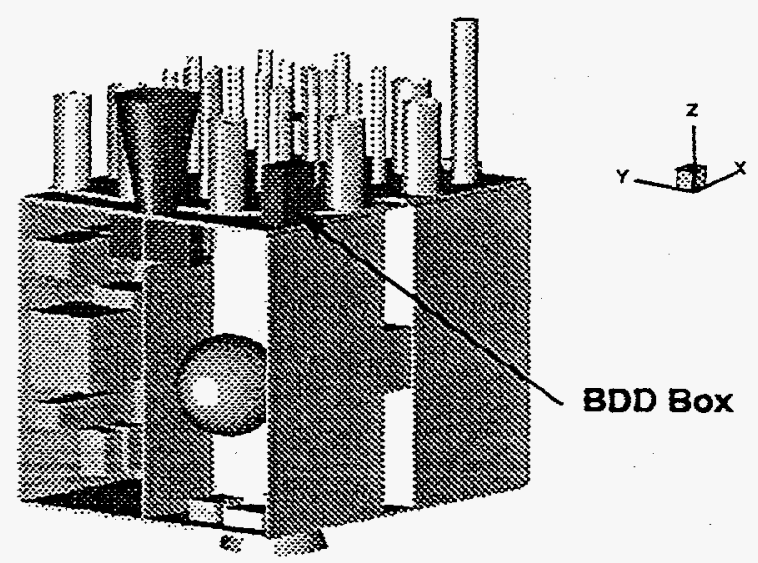

FIGURE 4. Approximate model of the GPS satellite obtained with BRI-CAD [8] showing the location of the BDD box.

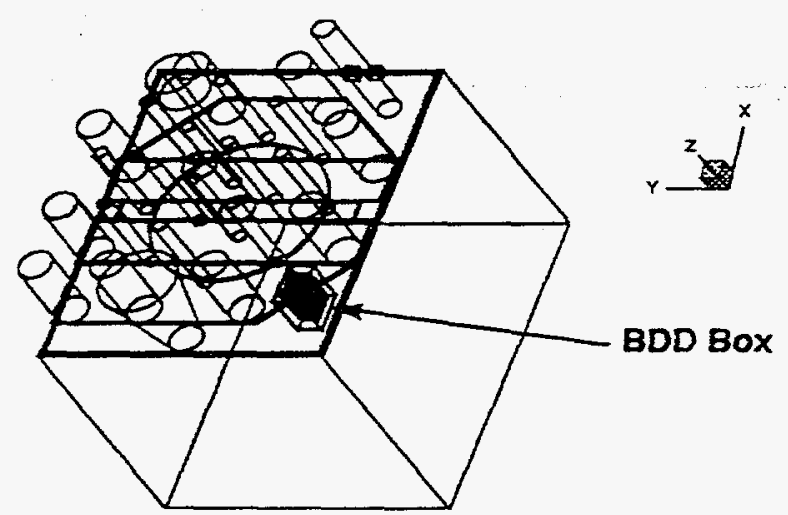

FTGURE 5. Projection of the combinatorial geometry body specification approximating the GPS satellite in simulations with the ACCEPT code of the MITS system.

The instrument is mounted on the outside of the satellite at the minimum- $Z$ plane of the projection of Fig. 3 . In the BRL$\mathrm{CAD}$ [8] model of Fig. 4, it is shown mounted atop the large, approximately rectangular parallelepiped (RPP) main body of the GPS satellite. Numerous other cylindrically shaped antennas and other components are similarly mounted atop the main body. All of these objects as well as the main body itself will perturb the natural environment, thus affecting the radiation field to which the BDD is exposed. Consequently, a realistic simulation of the effect of the natural environment on the BDD box should include the full satellite geometry.

What we have done in the present simulation is to include reasonably full detail of the BDD box itself as shown in Fig. 3, along with a simplified model of the rest of the satellite as already approximated in Fig. 4. The BRL-CAD model is already consistent with the CG specification of the ACCEPT code. We homogenized the main body as a single RPP and each of the cylindrical and conical features of Fig. 4 , as well as a few other less obvious features of that figure. They were homogenized to solid regions of aluminum with mass densities that preserve the total masses and external dimensions. The projection of the resulting CG body specification is plotted in Fig. 5 from a different perspective. The virtually black internal portion of the BDD box in the projection is a consequence of the detail to which this subsystem has been modeled (see Fig. 3).

\section{B. Source Specification}

Any non-reentrant surface element in a uniform isotropic radiation field will experience a constant inward-directed surface current (ignoring the half of the isotropic flux that makes a negative contribution to the current) that has a cosinelaw angular distribution with respect to the inward normal. Consequently, in forward mode we surround the satellite geometry with a non-reentrant surface, sample source positions uniformly in area over this surface, and sample source direction from a cosine-law distribution with respect to the local inward normal at the sampled source position, ignoring the outwardgoing half of the flux. On the one hand, the surface should circumscribe the satellite as snugly as possible for efficient sampling of source particles (minimize sampling of particles that miss the satellite). On the other hand, the surface should be simple enough to avoid unnecessarily complicated sampling algorithms. Any of the CG primitive body types of the ACCEPT code should satisfy the latter condition. We chose to surround the GPS satellite with a tightly fitting RPP, the source RPP. To obtain dose per uxit flux for absolute normalization, the default dose per source electron is multiplied by one fourth the area of the source RPP. A factor of one half comes from the ratio. of the $2 \pi$ steradian current density to the $2 \pi$ flux and the second factor of one half comes from the fact that the outwardgoing half of the flux is ignored in that calculation.

The space/angle sampling just described is carried out explicitly only for forward Monte Carlo calculations. In adjoint mode this sampling is replaced by an implicit folding of the adjoint flux based on the response function of the detector dose with the GPS uniform isotropic flux at the source RPP as shown in Eq. (2) of Ref. [2].

The spectrum of the uniform isotropic flux to which the six faces of the source RPP enclosing the satellite are exposed is 


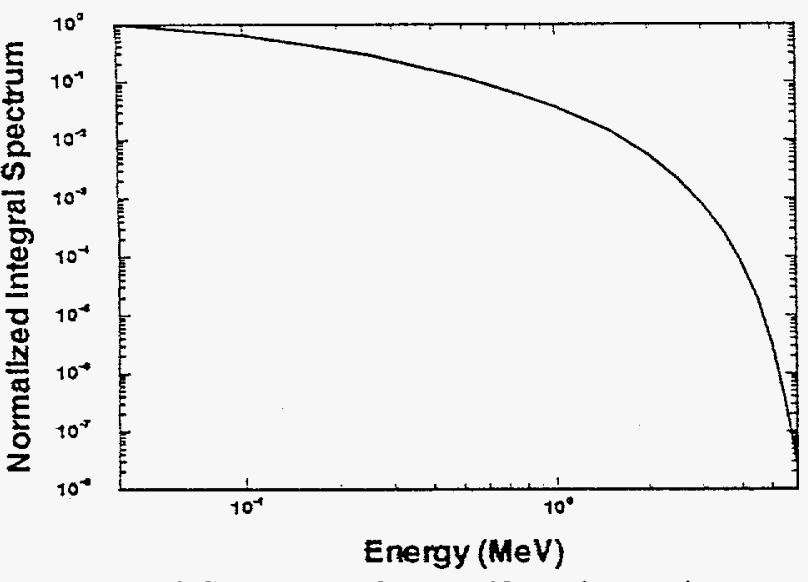

FIGURE 6. Spectrum of the uniform isotropic electron energy flux $[9,10]$ to which the GPS satellite is exposed, with minimum and maximum energies of 0.04 and $6.0 \mathrm{MeV}$, respectively.

plotted in Fig. $6[9,10]$. The minimum and maximum energies are 0.04 and $6.0 \mathrm{MeV}$, respectively. The thickness of the five external walls is more than the range of an electron with an energy of $2.0 \mathrm{MeV}$. Only about half a percent of the source electrons have energies greater than this value. Consequently, the source spectrum is dominated by electrons that do not have energies sufficient to penetrate the walls, and the dose is dominated by the effects of bremsstrablung radiation.

\section{Results}

In principle, a great deal of information can be obtained from a single adjoint calculation. Ultimately, of course, we want a single number, the dose at the selected location in the BDD box due to the isotropic radiation field described above. However, we can obtain this dose differential in the phasespace parameters of the source. This information may have many uses.

Consider, for example, the following. The source as we have described it is already uniform in space and direction. If wo were to make it uniform in energy as well (flat spectrum), wo could use the results to obtain the dose for any source within the energy range of the calculation. We would simply save the dose differential in all of the phase-space parameters. We could then obtain the dose for an arbitrary source after the MITS calculation has been completed by folding that source with the saved differential distributions. The numerical accuracy of this procedure would be limited only by the histogram form and the statistical accuracy of the distributions.

Perhaps more important is the possibility of employing the differential dose information for improving the design of the system. These distributions, or importance functions, reveal those source parameters that contribute most strongly to the calculated dose. They could point to minor rearrangements in subsystems or the optimization (i. e., minimization of weight penalty) of additional shielding that could significantly reduce radiation dose where necessary. Of course, such analyses are most effectively carried out during the design phase of a system.

Finally, the importance function from the adjoint calculation can be used to reduce the variance of a forward calculation. Suppose, for example, that point deposition is not sufficient, but rather a localized dose profile is required. Obtaining this profile would necessitate multiple adjoint calculations. On the other hand, the importance function from a single adjoint calculation of the dose in the region of interest could be employed to efficiently bias. a single forward calculation of the dose profile. It should be emphasized, however, that the profile must be sufficiently localized that the importance function is valid for the entire profile region.
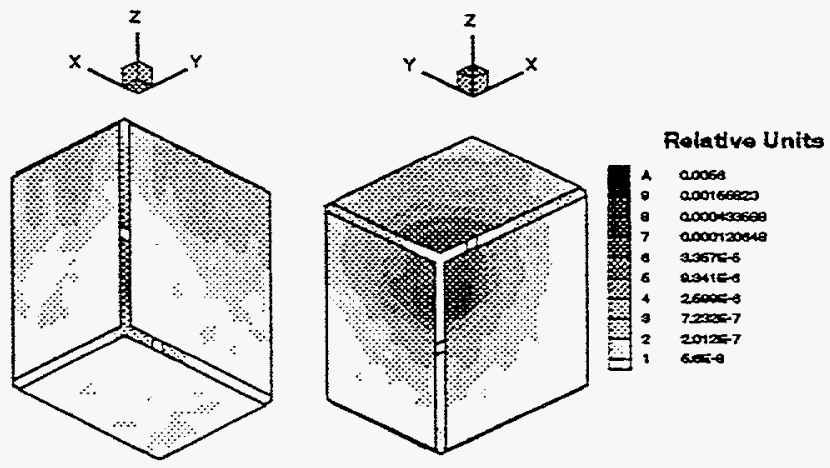

FIGURE 7. Contour plots of the spatial importance function of the detector dose using a flat spectrum of source electrons from 0.04 to $6.0 \mathrm{MeV}$.

Figure 7 shows, from two perspectives, a pair of contour plots of the dose to the specified detector in the BDD box differential in source position over the surface of the source RPP. In this case, however, we have employed a flat source spectrum from 0.04 to $6.0 \mathrm{MeV}$ instead of the GPS spectrum. These exponential contours of the spatial importance function show the dominance of the source near the box as indicated by the dark region in the comer of the minimum-Y plane. Had we chosen a detector location deeper within the satellite, the

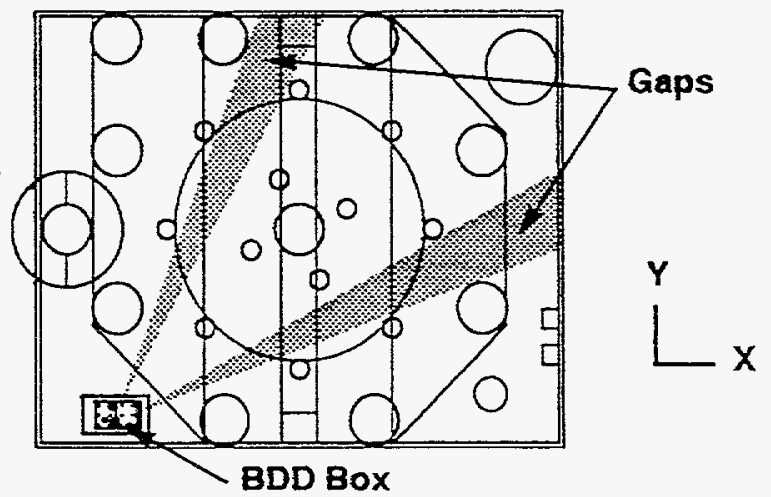

FIGURE 8. Projection of the GPS body specification showing two unobstructed paths from the maximum- $X$ and maximum-Y surfaces of the source RPP to the BDD box that correlate with regions of high spatial importance on these surfaces in Fig. 7. 
dynamic range of this function would presumably have been much smaller, and the dose would not have been dominated by so small a spatial region of the source.

Note the elongated dark regions on the maximum- $\mathrm{X}$ and maximum- $Y$ planes near the top of satellite and away from the BDD box. These correspond to source areas of these planes where the radiation has a relatively unobstructed path to the box. They can be correlated with gaps within the antenna field as shown in Fig. 8. If this were a significant contribution to the dose, designers could, for example, use this information to rearrange the antenna field and/or add shielding in such a way as to minimize the weight penalty.

In Fig. 9a, we have plotted the contribution to the dose from the minimum-Y plane (i.e., the one that dominates the total dose) differential in source energy, as well as its integral, for the flat source spectrum. This energy importance function shows the dominance of high-energy source electrons for a flat source spectrum. This dominance may be due to the fact that highenergy electrons have the best chance of reaching the detector and/or that they produce more radiation that can reach the detector. The statistical uncertainty in this contribution was $0.28 \%$ for a run time of about $20 \mathrm{hrs}$.

Figure $9 \mathrm{~b}$ shows the same importance function for the GPS spectrum. The dominance of low-energy electrons in the spectrum is reflected in their increased importance here. However, the large statistical fluctuations lead to a statistical uncertainty in the contribution of this source plane to the total dose that is $5.3 \%$ for a nun time of 20.9 brs. The large statistical uncertainty relative to the flat source spectrum is a consequence of the fact that while Fig. 9a shows that low-energy source electrons make a disproportionately low (statistically poor) contribution to the dose, it is just these low-energy electrons that are highly weighted by the GPS spectrum.

Thus, even in adjoint transport, variance reduction is highly desirable, if not necessary. Because the detector is beyond the range of all but a few of the source electrons, we are faced with a combined radiation effects calculation. Such simulations are among the most difficult for Monte Carlo electron-photon transport, forward or adjoint. This is because variancereduction methods for the efficient calculation of the production of radiation by electrons generally impede those for efficiently calculating the energy deposition resulting from that radiation.

To overcome this difficulty, we make use of a new variancereduction package [11] that will bo implemented in both MITS and ITS. In particular, for the present adjoint calculation variance reduction was achieved by biasing electron-to-photon cross sections in the vicimity of the source and photon-toelectron production in the vicinity of the detector. The resulting energy importance function for the GPS spectrum corresponding to Fig. $9 \mathrm{~b}$ is plotted in Fig. 9c. Except for an anomaly near $1.0 \mathrm{MeV}$, the biasing has substantially reduced the statistical fluctuations. The run time was $9.42 \mathrm{hrs}$, and we

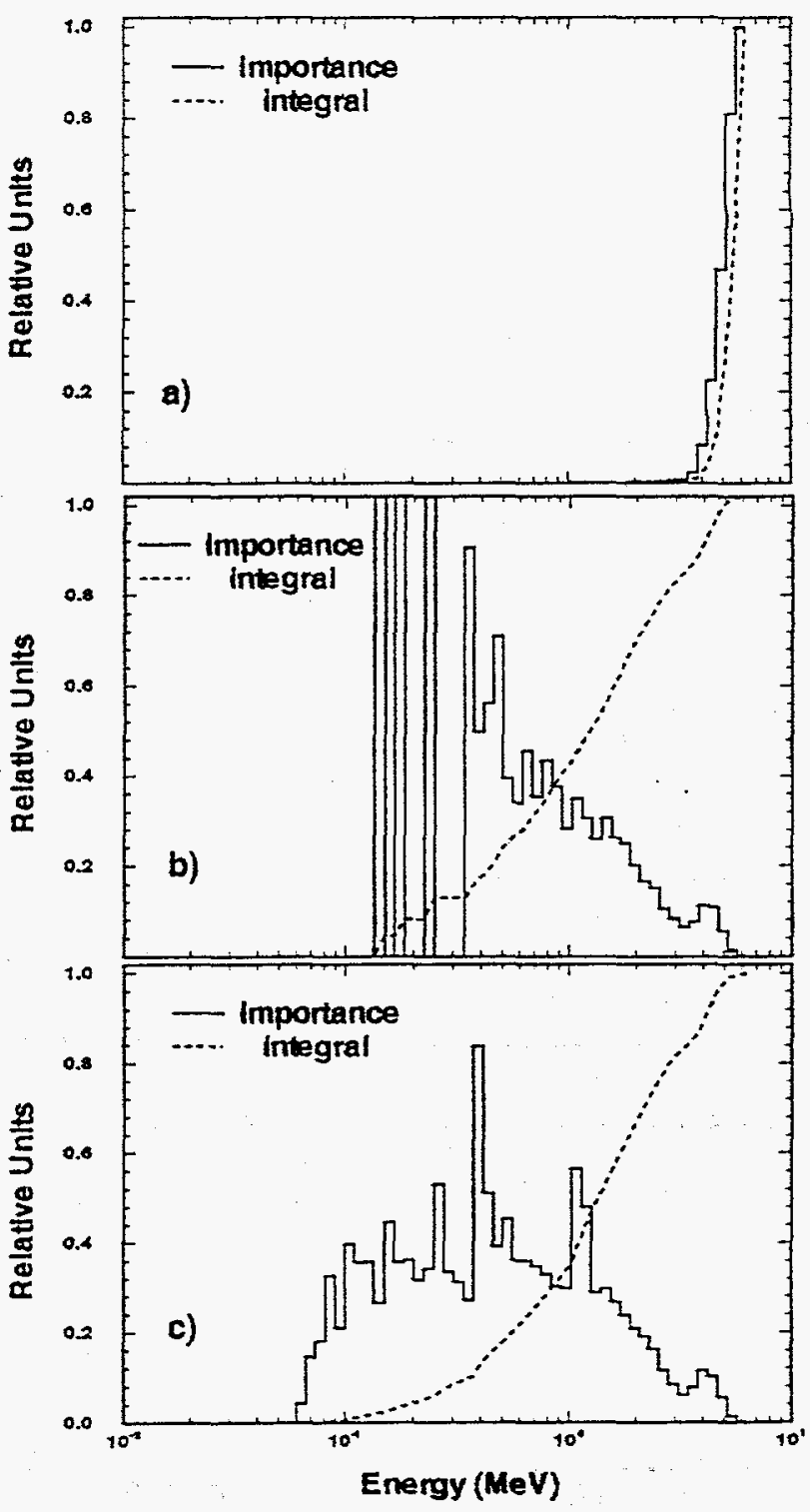

FIGURE 9. Energy importance functions of the detector dose and their integrals for the minimum-Y plane of the source RPP for (a) the flat spectrum without variance reduction, (b) the GPS spectrum without variance reduction, and (c) the GPS spectrum with variance reduction.

obtain $2.21 \times 10^{1 \mathrm{~V}} \mathrm{MeV} / \mathrm{g}-\mathrm{yr}$ as the contribution to the dose from this surface with a statistical uncertainty of $4.4 \%$. The biasing has reduced the variance by a factor of three (Eq. (5) of Ref. [2]). The contribution to the dose without biasing was $2.06 \times 10^{10} \mathrm{MeV} / \mathrm{g}-\mathrm{yr}$, which agrees with the biased result within the statistical uncertainties.

The total dose to the detector for the GPS environment, as well as the contribution to the dose from each of the six sides of the source RPP, are shown in Table 1. Results are shown with and without the use of the variance reduction package. The numbers in parentheses are the estimated one-sigma statistical uncertainties expressed as a percent of the dose that they follow. About half the total dose comes from the minimum-Y surface. 
That surface together with the minimum- $X$ and maximum- $Z$ surfaces contribute over $90 \%$ of the total because these planes offer the most unobstructed exposure of the BDD box to the electron environment. Still, probably in excess of $5 \%$ is contributed by the other 3 planes. This distribution of the dose among the source planes is, of course, unique to the detector we have chosen. A detector somewhere else in the satellite system will, in general, have an entirely different spatial importance function.

\begin{tabular}{|l|ll|ll|}
\cline { 2 - 5 } \multicolumn{1}{c|}{} & \multicolumn{4}{c|}{ Dose (MeV/g-yr) } \\
\hline surface & no variance reduction & variance reduction \\
\hline $\min y$ & $2.06 \times 10^{10}$ & $(5.3)$ & $2.21 \times 10^{10}$ & $(4.4)$ \\
\hline $\max y$ & $1.60 \times 10^{9}$ & $(18)$. & $1.49 \times 10^{9}$ & $(20)$. \\
\hline $\min x$ & $7.78 \times 10^{9}$ & $(10)$. & $7.80 \times 10^{9}$ & $(6.5)$ \\
\hline $\max x$ & $1.12 \times 10^{9}$ & $(16)$. & $6.83 \times 10^{8}$ & $(8.6)$ \\
\hline $\min z$ & $1.34 \times 10^{8}$ & $(11)$. & $1.48 \times 10^{8}$ & $(14)$. \\
\hline $\max z$ & $6.70 \times 10^{9}$ & $(11)$. & $8.98 \times 10^{9}$ & $(11)$. \\
\hline total & $3.78 \times 10^{10}$ & $(4.2)$ & $4.13 \times 10^{10}$ & $(3.7)$ \\
\hline
\end{tabular}

TABLE 1: Total dose to the detector for the GPS environment and contribution to the dose from each of the six sides of the source RPP.

\section{SUMMMARY AND CONCLUSIONS}

Using the MITS system, we have demonstrated the exceptional efficiency of adjoint Monte Carlo for simulating the responses of point detectors in space applications.

Dose predictions of the ACCEPT code of the MITS code system in the adjoint mode are in very good agreement with the three dimensional measurements of Van Gunten from an experimentally simulated uniform isotropic electron flux. It would be desirable to have such data for wall thicknesses beyond the ranges of the source electrons. Predicting these radiation-dominated doses would be a more severe test of the MITS codes.

In the much more complex simulation of the dose to a detector in the GPS satellite, the MITS system was able to generate spatial and energy importance functions. These are powerful tools for analyzing the radiation survivability of space systems. Moreover, they can be employed to predict tho dose to the detector for an arbitrary source distribution within the phase space spanned by the calculation.

Much work remains to be done in order to optimize variance reduction procedures for the adjoint mode of the MTTS system and to efficiently use adjoint results for optimizing forward Monte Carlo simulations.

The GPS application is an application where not only are run times of the forward solutions very long, if not prohibitive, but the adjoint solutions themselves require very long run times as well. Extensive analyses of these applications will require parallel processing. There are already developmental parallel versions of the ITS systems that have been run on a UNIX workstation network[12], the Intel PARAGON [13], and both few-processor [14] and many-processor (Maui High Perfomance Computing Center) [15] IBM SP2 systems. Because Monte Carlo is inherently parallelizable, this work shows that efficiencies in excess of $90 \%$ aro easily achieved for large problems. Version 4.0 of the ITS system will have a parallel option. This capability is easily extendible to MUTS as well. Moreover, achieving a capability for running either code system on the multi-teraflop machines now being developed should bo straightforward.

\section{ACKNOWLEDGMENTS}

We gratefully acknowledge C. David Turner, Wesley C. Fan, and Stanley B. Roeske for their advice throughout this work and for providing information on the geometry and radiation environment of the GPS satellite. This work was supported by the U.S. Department of Energy under Contract No. DE-AC04-94AL8500.

\section{REFERENCES}

[1] J. A. Halbleib, R. P. Kensek, T. A. Mehlhom, G. D. Valdez, S.M. Seltzer, and M. J. Berger, "ITS: The Integrated TIGER Series of Electron/Photon Codes - Version 3.0, "IEEE Trans. Nikcl Sci, Vol. 39, 1025 (1992).

[2] L. J. Lorence, R. P. Kensek, J. A. Halbleib, and J. E. Morel, "Adjoint Electron-Photon Transport Monte Cario Calculations with ITS," LEEE Trans. Nucl Sci, Vol. 42, No. 6, December 1995, 1895-1901 (1995).

[3] L. J. Lorence, Jr, J. E. Morel, and G. D. Valdez, "Results Guide to CEPXS/ONELD: A One-Dimensional Coupled ElectronPhoton Discrete Ordinates Code Pacikage, Version 1.0," SAND89-22111, Sandia National Laboratories (1990).

[4] J. E. Morel, L. J. Lonence, R. P. Kensek, J. A. Halbleib, and D. P. Sloan, "A Hybrid Multigroup/Continuous-Energy Monte Carlo Method for Solving the Boltzmann-Fokker-Planck Equation," accepted for publication in NucL Sci and Eng.

[5] 0.0. Van Gunten, "Three Dimensional Electron Dose Distribution Measurements," Ph. D. Dissertation, 1974, University of Maryland, University Microfilms, Ann Arbor, Michigan.

[6] O. O. Van Gunten, R. E. Starrett, and V. G. Adams, "Development of a Ground-Based Facility for Simulating the Omnidirectional Near-Earth Electron Environment," NucL Irstr. Meth. in Phys. Res., Vol. B51, 173-181 (1990). 
[7] In Monte Carlo parlance the region where the dose is calculated is called a "detector" region. We henceforth use the term detector to denote such a region.

[8] "The Ballistic Research Laboratory CAD Package Release 4.0," Advanced Computing Systems, The U.S. Amy Ballistic Research Laboratory, Aberdeen Proving Ground, Maryland 21005-5066, December 1991.

[9] "AE-8" undocumented data set distributed by the National Space Science Data Center, NASA Goddard Space Flight Center.

[10] "SPACE RADIATION User's Manual," Sevem Communications Corporation, 1990.

[11] J. A. Halbleib, R. P. Kensek, and G. D. Valdez, "Variance Reduction Unique to Coupled Electron/Photon Monte Carlo Transpont," Advanced Monte Carlo Computer Programs for Radiation Transport, April 27-29, 1993, Saclay, France, NEA OECD, Saclay, France, ISBN 92-64-14376-9, 141-153.

[12] W. C. Fan and J. A. Halbleib, "Distributed Multitasking ITS with PVM," Trans. Am Nucl Soc., Vol. 72, 146-147 (1995).

[13] W. C. Fan, C. D. Tumer, J. A. Halbieib, Sr., and R. P. Kensek, "Recent Development for the ITS Code System - Parallel Processing and Visualization," Proceedings of the 1996 Topical Meeting on Radiation Protection and Shielding, No. Falmouth, Massachusetts, April 21-25, 1996, 79-85 (1996).

[14] G.D. Valdez, "Parallelization of ITS on an IBM SP2 Computer," Trans. Am Nucl Soc. Vol. 73, 331-332 (1995).

[15] G. D. Valdez, private communication, Sandia National Laboratories, 1996.

\section{DISCLAIMER}

This report was prepared as an account of work sponsored by an agency of the United States This report was prepared as an account of work sponsor any agency thereof, nor any of their Government. Neither the United States Government nor any agency theral liability or responsiemployees, makes any warranty, express or implied, or assumes any legal liability or roduct, bility for the accuracy, completeness, or usefulness of any information, apparatus, product, process disclosed, or represents that its use would not infringe privately ow right ence herein to any specific commercial product, process, or service by trade name, trademark manufacturer, or otherwise does not necessarily constitute or imply its endorem mendation, or favoring by the United States Government or any agency thereor. The views and opinions of authors expressed herein do not necessarily state or reflect those of the United States Government or any agency thereof. 
